\title{
Growth Regulation of Bahiagrass (Paspalum notatum Fluegge) with Imazaquin and $A C$ 263,222
}

\author{
J. Michael Goatley, Jr. ${ }^{1}$, and Victor L. Maddox ${ }^{2}$ \\ Department of Plant and Soil Sciences, Mississippi State University, Mississippi \\ State, MS 39762
}

\author{
Robert M. Watkins ${ }^{3}$ \\ American Cyanamid Company, Starkville, MS 39759
}

Additional index words. bahiagrass, seedhead suppression, vegetative suppression, imidazolinones

\begin{abstract}
Research was conducted over 2 years to evaluate efficacy of various levels and combinations of imazaquin and $A C 263,222$ for growth regulation of unimproved bahiagrass ('Pensacola') turf. Imazaquin at $0.42 \mathrm{~kg} \cdot \mathrm{ha}^{-1}$ caused only slight bahiagrass discoloration in all trials and reduced seedhead count (as compared to the nontreated control) by $\geq 80 \%$ for 8 weeks after treatment (WAT) following a July 1992 application. AC 263,222 at 0.042 or $0.056 \mathrm{~kg} \cdot \mathrm{ha}^{-1}$ applied in late May or June provided $100 \%$ seedhead control through 8 WAT. However, AC 263,222 applied in July 1992 and Aug. 1993 at 0.056 kg·ha ${ }^{-1}$ resulted in unacceptable discoloration through 8 WAT. AC 263,222 at 0.014 or $0.028 \mathrm{~kg} \cdot \mathrm{ha}^{-1}$ provided $\geq 90 \%$ seedhead control with only minimal discoloration following applications in July or Aug. 1993, indicating that lower rates of AC 263,222 provided acceptable seedhead control of bahiagrass during times when growth was slowed due to moisture stress. Chemical names used: $( \pm)-2$-[4,5-dihydro-4-methyl-4-(1-methylethyl)-5-oxo-1 Himidazol-2-yl]-5-methyl-3-pyridinecarboxylic acid (AC 263,222); 2-[4,5-dihydro-4methyl-4-(1-methylethyl)-5-oxo-1 $H$-imidazol-2-yl]-3-quinolinecarboxylic acid (imazaquin).
\end{abstract}

Bahiagrass is a popular low-maintenance turfgrass used for home lawns and unimproved turf areas in the southern Gulf state regions of the United States. A major maintenance problem for bahiagrass turf is its propensity for rapid seedhead production throughout the growing season (Beard, 1973). Seedheads emerge quickly after mowing and the flowering culm can expand to heights of 70 to $80 \mathrm{~cm}$, thus reducing turf quality, increasing mowing frequency, and posing a hazard to motorists by reducing visibility in highway rights-of-way (Busey, 1985).

Suppression of vegetative and reproductive growth of bahiagrass with plant growth regulators (PGR) reduces mowing frequency, consequently saving time and money (Johnson, 1990). Concern over turfgrass injury continues to limit widespread PGR use (Flanagan and Peacock, 1986; Johnson, 1990; Watschke et al., 1992). Treatments with $S$-ethyl dipropyl thiocarbamate (EPTC), EPTC + 3,6-dichloro2-methoxybensoic acid (dicamba), 2[1(ethoxyimino)butyl]-5-[2-(ethylthio)propyl]3-hydroxy-2-cyclohexen-1-one (sethoxydim), and sethoxydim $+N$-[2,4-dimethyl-5[ [ trifluoromethy 1$)$ - sulfon y l]

Received for publication 19 Oct. 1995. Accepted for publication 17 Mar. 1996. Paper no. J-8746 of the Mississippi Agricultural Forestry Experiment Station. The cost of publishing this paper was defrayed in part by the payment of page charges. Under postal regulations, this paper therefore must be hereby marked advertisement solely to indicate this fact. ${ }^{1}$ Associate Professor.

${ }^{2}$ Research Assistant.

${ }^{3}$ Senior Research Agriculturist. amino]phenyl]acetamide (mefluidide) suppressed bahiagrass growth on Florida highway rights-of-ways with limited turfgrass damage (Flanagan and Peacock, 1986). In addition, bahiagrass seedhead suppression followed three consecutive applications of either $\alpha$ ( m e t h y le th y 1 ) - $\alpha-$ [ 4 (trifluoromethoxy)phenyl]-5-pyrimidine methanol (flurprimidol) at $0.56 \mathrm{~kg} \cdot \mathrm{ha}^{-1}$ or mefluidide at $0.56 \mathrm{~kg} \cdot \mathrm{ha}^{-1}$ applied at 4-week intervals. 2-[4,5-Dihydro-4-methyl-4-(1methylethyl)-5-oxo-1 $H$-imidazol-2-yl]-5ethyl-3-pyridinecarboxylic acid (Imazethapyr), glyphosate-isopropylamine salt [isopropylamine salt of $N$-(phosphonomethyl) glycine], and glyphosate-sodium sesqui salt [sodium sesqui salt of $N$-(phosphonomethyl) glycine] plus (2,4-dichlorophenoxy)acetic acid (2,4-D) severely injured bahiagrass for 4 to 6 weeks after treatment (WAT) but controlled seedheads for 10 WAT without permanently reducing turf density (Johnson, 1990).

Imidazolinones, applied postemergence, control numerous broadleaf weeds and have shown potential for growth regulation of warmseason turfgrasses (DiPaola et al., 1985; Goatley et al., 1993; McCarty et al., 1993). This research was conducted to determine the efficacy of various rates and combinations of imazaquin and AC 263,222 (AC) on color, seedhead development, and vegetative growth of bahiagrass.

\section{Materials and Methods}

Experiments were conducted on established, unimproved bahiagrass that had re- ceived no prior herbicide treatment. Treatment dates were 19 May, 26 June, and 23 July 1992, and 31 May, 28 June, 26 July, and 25 Aug. 1993. In 1992, May and June treatments were made on a farm in north-central Mississippi (Prentiss silt loam; coarse-loamy, thermic, siliceous Ochreptic Fragiudults; $\mathrm{pH}$ 5.8). The July treatment was applied at the Plant Science Research Center at Mississippi State Univ. (Marietta fine sandy loam; fine-loamy, mixed, thermic, siliceous Aquic Fluventic Eutrochrepts; $\mathrm{pH} 6.4)$. The monthly treatments in 1993 were made on a farm in east-central Mississippi (Savannah fine sandy loam; fineloamy, thermic, siliceous Typic Fragiudults; $\mathrm{pH}$ 5.9).

In 1992, May treatments were $\left(\mathrm{kg} \cdot \mathrm{ha}^{-1}\right)$ imazaquin, 0.42; AC, 0.056; and imazaquin (0.21) plus AC (0.056). In June, treatments were expanded to include AC (0.028) and imazaquin (0.21) plus AC (0.028). July treatments were the same as in June, except the lowest rate of AC (0.028) was replaced with 0.042 and applied either alone or combined with imazaquin at 0.21 . In 1993, all monthly treatments were AC at $0.014,0.028$, and 0.056 $\mathrm{kg} \cdot \mathrm{ha}^{-1}$.

The PGRs were applied in a water carrier by means of a $\mathrm{CO}_{2}$-pressurized backpack sprayer calibrated to deliver the aqueous carrier at $560 \mathrm{~L} \cdot \mathrm{ha}^{-1}$ and $200 \mathrm{kPa}$. Plots were 1.8 $\times 3.0 \mathrm{~m}$ in 1992 and $1.8 \times 6.0 \mathrm{~m}$ in 1993 . No fertilizer or supplemental irrigation were used at either location. Plots were clipped to a height of $12 \mathrm{~cm} 1$ day before treatment in 1992 and 3 days before treatment in 1993 .

Data were collected at 2-week intervals up to 8 WAT (only 6 WAT for the 25 Aug. 1993 treatment due to early frost) for bahiagrass color, seedhead count and height, and vegetative canopy height. Turfgrass color was visually rated on a scale of 1 to $9(1=$ completely brown turf, $6=$ minimal acceptable color, and $9=$ dark green turf). Seedhead counts were determined by tossing a $3-\mathrm{dm}^{2}$ circular template at three random locations within each plot, counting seedheads within the template, and averaging. Vegetative canopy heights and seedhead heights also were measured at the three random locations within each plot, and the values were averaged.

Percent reductions in seedhead counts and heights, and canopy heights were calculated by dividing the value for the respective variable measured for the imidazolinone treatments by the value for the same variable for the nontreated control for a given rating period and subtracting the quotient from 100 .

Treatments in each experiment were arranged in a randomized complete-block design with three replications in 1992 and four in 1993. Data were analyzed using the General Linear Models (GLM) procedure of SAS for analysis of variance (ANOVA) (SAS Institute, 1987). Mean separation for 1992 data was done with Fisher's Protected LSD procedure, when appropriate. Gradient trend analysis (Lentner and Bishop, 1986) was used to examine treatment means for linear, quadratic, or cubic trends for the 1993 data, when appropriate. 


\section{Results and Discussion}

Turfgrass color. Imazaquin and AC lowered color ratings, relative to the control, through 4 WAT after the May 1992 application. However, these treatments did not impair color at 6 and 8 WAT (Table 1). Imazaquin at $0.42 \mathrm{~kg} \cdot \mathrm{ha}^{-1}$ in June caused some discoloration at 2 WAT, but all color ratings were equal by 8 WAT (Table 1). Following the June 1992 application, all AC-treated turf exhibited significantly poorer color than the control through 6 WAT, although color ratings for all treatments were acceptable $(\geq 6)$. Following July applications, $\mathrm{AC}$ at all rates and combinations impaired turf color through 8 WAT. AC at $0.056 \mathrm{~kg} \cdot \mathrm{ha}^{-1}$ alone or combined with imazaquin resulted in unacceptable turf color at 8 WAT (Table 1)

Color response trends to AC in 1993 were quadratic through 6 WAT in May and 4 WAT in June, with $0.056 \mathrm{~kg} \cdot \mathrm{ha}^{-1}$ causing the most turf discoloration in both months (Table 2). Quadratic trends indicated little difference in color ratings between the control and $\mathrm{AC}$ at $0.014 \mathrm{~kg} \cdot \mathrm{ha}^{-1}$ on any rating date, whereas turf color was poorer in plots treated with $\mathrm{AC}$ at 0.028 or $0.056 \mathrm{~kg} \cdot \mathrm{ha}^{-1}$. No differences in color were evident at 8 WAT in May and 6 WAT in June. Both linear and quadratic trends existed through 4 WAT in July, again indicating less discoloration for $\mathrm{AC}$ at $0.014 \mathrm{~kg} \cdot \mathrm{ha}^{-1}$ as compared to higher rates (Table 2). However, the linear trend indicated a slight decline in color for $0.014 \mathrm{~kg} \cdot \mathrm{ha}^{-1}$ as compared to the control. Discoloration was not detected by 6 WAT in July. Color ratings were lower for all AC treatments through 4 WAT in August, although ratings for $0.014 \mathrm{~kg} \cdot \mathrm{ha}^{-1}$ were never unacceptable. AC at $0.056 \mathrm{~kg} \cdot \mathrm{ha}^{-1}$ resulted in unacceptable color at 4 and 6 WAT in Aug. 1993 (Table 2).

Seedhead count and height. All concentrations of AC completely suppressed seedhead emergence through 8 weeks after the May 1992 application (Table 3). Imazaquin alone at $0.42 \mathrm{~kg} \cdot \mathrm{ha}^{-1}$ provided $100 \%$ seedhead control through 4 WAT and a $72 \%$ reduction in seedheads as compared to the control by 8 WAT (Table 3). In June, all AC concentrations plus imazaquin or $\mathrm{AC}$ alone at 0.056 $\mathrm{kg} \cdot \mathrm{ha}^{-1}$ provided $\geq 95 \%$ seedhead control through 8 WAT. Imazaquin at $0.42 \mathrm{~kg} \cdot \mathrm{ha}^{-1}$ reduced seedhead count by $54 \%$, and $\mathrm{AC}$ at $0.028 \mathrm{~kg} \cdot \mathrm{ha}^{-1}$ reduced seedhead count by $88 \%$ as compared to the control at $8 \mathrm{WAT}$ in June. For the July application, all treatments containing AC provided $\geq 99 \%$ control of seedheads through 8 WAT, while imazaquin alone at $0.42 \mathrm{~kg} \cdot \mathrm{ha}^{-1}$ resulted in $\geq 90 \%$ control of bahiagrass seedheads 8 WAT.

$\mathrm{AC}$ at $0.014 \mathrm{~kg} \cdot \mathrm{ha}^{-1}$ reduced bahiagrass seedhead counts by $43 \%, 36 \%, 87 \%$, and $87 \%$ at 8 WAT for the May through Aug. 1993 treatments, respectively (Table 4). This result again indicated that the lower concentrations of AC were sufficient for bahiagrass seedhead control later in the growing season. $\mathrm{AC}$ at $0.028 \mathrm{~kg} \cdot \mathrm{ha}^{-1}$ provided $\geq 87 \%$ seedhead control as compared to the control at 8 WAT for the duration of each experiment. The 0.056 $\mathrm{kg} \cdot \mathrm{ha}^{-1}$ rate completely suppressed seedhead emergence through 8 WAT following May and July applications, and had $\geq 95 \%$ success through completion of the trials initiated in June and August (Table 4).

Trends in seedhead height reduction were similar to those reported for seedhead counts. Imazaquin at $0.42 \mathrm{~kg} \cdot \mathrm{ha}^{-1}$ reduced seedhead height from $35 \%$ to $91 \%$ as compared to the control at 8 WAT from May to July 1992, again indicating that the PGR activity of these imidazolinones was greater as bahiagrass growth rates slowed later in the season (Table 5). All treatments containing AC at rates $\geq 0.028$ $\mathrm{kg} \cdot \mathrm{ha}^{-1}$ provided $\geq 88 \%$ seedhead height reductions at 8 WAT.

In 1993, seedhead height reduction for AC treatments followed linear, quadratic, and cubic trends through the final rating date of each monthly trial (Table 6). These trends indicate that 0.028 and $0.056 \mathrm{~kg} \cdot \mathrm{ha}^{-1}$ more effectively reduced seedhead height than $0.014 \mathrm{~kg} \cdot \mathrm{ha}^{-1}$. Seedhead height reductions with AC at 0.014 $\mathrm{kg} \cdot \mathrm{ha}^{-1}$ were $15 \%$ and $5 \%$, respectively, at 8 WAT following May and June applications, and increased to $35 \%$ and $40 \%$ at 8 WAT following July and August applications. In May $19930.056 \mathrm{~kg} \cdot \mathrm{ha}^{-1}$ provided $100 \%$ seedhead control through 8 WAT and 0.028 $\mathrm{kg} \cdot \mathrm{ha}^{-1}$ resulted in a $40 \%$ reduction in seedhead height as compared to the control (Table 6). For the June, July, and Aug. 1993 treatment dates, the largest reductions in seedhead height at 8 WAT were with $\mathrm{AC}$ at $0.056 \mathrm{~kg} \cdot \mathrm{ha}^{-1}$. However, height reductions provided by 0.028 $\mathrm{kg} \cdot \mathrm{ha}^{-1}$ were within $10 \%$ of those recorded for $0.056 \mathrm{~kg} \cdot h \mathrm{a}^{-1}$ by the end of each trial (Table 6).

Vegetative canopy height. Imazaquin at $0.42 \mathrm{~kg} \cdot \mathrm{ha}^{-1}$ provided $\geq 40 \%$ reduction in bahiagrass vegetative canopy height as com-

Table 1. Bahiagrass color ratings at 2, 4, 6, and 8 weeks after treatment (WAT) following imidazolinone [Imazaquin (I); AC 263,222 (AC)] applications in May, June, and July 1992.

\begin{tabular}{|c|c|c|c|c|c|c|c|c|c|c|c|c|c|}
\hline \multirow[b]{5}{*}{ Imidazolinone } & \multirow{5}{*}{$\begin{array}{c}\text { Concn } \\
\left(\mathrm{kg} \cdot \mathrm{ha}^{-1}\right)\end{array}$} & \multicolumn{12}{|c|}{ Turf color (rating) ${ }^{2}$} \\
\hline & & \multicolumn{12}{|c|}{ Application date } \\
\hline & & \multicolumn{4}{|c|}{19 May } & \multicolumn{4}{|c|}{26 June } & \multicolumn{4}{|c|}{23 July } \\
\hline & & & & & & & & & & & & & \\
\hline & & 2 & 4 & 6 & 8 & 2 & 4 & 6 & 8 & 2 & 4 & 6 & 8 \\
\hline $\bar{I}$ & 0.42 & $5.5 \mathrm{~b}^{\mathrm{y}}$ & $5.5 \mathrm{~b}$ & 7.0 & 7.0 & $6.3 \mathrm{~b}$ & $6.8 \mathrm{a}$ & $7.0 \mathrm{a}$ & 7.0 & $5.2 \mathrm{~b}$ & $6.0 \mathrm{~b}$ & $6.8 \mathrm{~b}$ & $7.0 \mathrm{a}$ \\
\hline \multirow[t]{3}{*}{$\mathrm{AC}$} & 0.028 & --- & --- & --- & --- & $6.3 \mathrm{~b}$ & $6.0 \mathrm{~b}$ & $6.0 \mathrm{c}$ & 7.0 & --- & --- & --- & --- \\
\hline & 0.042 & --- & --- & --- & --- & --- & --- & --- & --- & $5.0 \mathrm{~b}$ & $5.8 \mathrm{bc}$ & $6.2 \mathrm{c}$ & $6.0 \mathrm{~b}$ \\
\hline & 0.056 & $5.0 \mathrm{c}$ & $4.8 \mathrm{c}$ & 6.8 & 7.0 & $6.2 \mathrm{~b}$ & $6.0 \mathrm{~b}$ & $6.0 \mathrm{c}$ & 7.0 & $5.0 \mathrm{~b}$ & $5.8 \mathrm{bc}$ & $6.0 \mathrm{~cd}$ & $5.5 \mathrm{c}$ \\
\hline \multirow[t]{3}{*}{$\mathrm{I}+\mathrm{AC}$} & $0.21+0.028$ & --- & --- & --- & --- & $6.0 \mathrm{~b}$ & $6.0 \mathrm{~b}$ & $6.5 \mathrm{~b}$ & 7.0 & --- & --- & --- & --- \\
\hline & $0.21+0.042$ & --- & --- & --- & --- & --- & --- & --- & --- & $5.0 \mathrm{~b}$ & $5.7 \mathrm{bc}$ & $6.2 \mathrm{c}$ & $5.8 \mathrm{~b}$ \\
\hline & $0.21+0.056$ & $5.0 \mathrm{c}$ & $4.8 \mathrm{c}$ & 6.8 & 7.0 & $6.3 \mathrm{~b}$ & $6.0 \mathrm{~b}$ & $6.0 \mathrm{c}$ & 7.0 & $5.0 \mathrm{~b}$ & $5.5 \mathrm{c}$ & $5.7 \mathrm{~d}$ & $5.5 \mathrm{c}$ \\
\hline Control & --- & $6.0 \mathrm{a}$ & $7.0 \mathrm{a}$ & 7.0 & 7.0 & $7.3 \mathrm{a}$ & $7.0 \mathrm{a}$ & $7.0 \mathrm{a}$ & 7.0 & $7.0 \mathrm{a}$ & $7.2 \mathrm{a}$ & $7.5 \mathrm{a}$ & $7.0 \mathrm{a}$ \\
\hline LSD & --- & 0.5 & 0.6 & NS & NS & 0.5 & 0.3 & 0.3 & NS & 0.4 & 0.4 & 0.4 & 0.2 \\
\hline
\end{tabular}

${ }^{2}$ Turfgrass color rating scale: $1=$ completely brown, $6=$ minimally acceptable green, $9=$ dark green .

${ }^{y}$ Mean separation within a column by Fisher's Protected LSD test at $P<0.05$; NS $=$ nonsignificant.

Table 2. Bahiagrass color ratings at 2, 4, 6, and 8 weeks after treatment (WAT) following AC 263,222 (AC) applications in May, June, July, and Aug. 1993.

\begin{tabular}{|c|c|c|c|c|c|c|c|c|c|c|c|c|c|c|c|}
\hline \multirow{5}{*}{$\begin{array}{l}\text { AC concn } \\
\left(\mathrm{kg} \cdot \mathrm{ha}^{-1}\right)\end{array}$} & \multicolumn{15}{|c|}{ Turf color $^{2}$} \\
\hline & \multicolumn{15}{|c|}{ Application date } \\
\hline & \multicolumn{4}{|c|}{31 May } & \multicolumn{4}{|c|}{28 June } & \multicolumn{4}{|c|}{26 July } & \multicolumn{3}{|c|}{25 Aug. } \\
\hline & & & & & & & & WAT & & & & & & & \\
\hline & 2 & 4 & 6 & 8 & 2 & 4 & 6 & 8 & 2 & 4 & 6 & 8 & 2 & 4 & 6 \\
\hline 0.014 & 7.0 & 7.6 & 7.0 & 6.6 & 7.0 & 7.0 & 7.3 & 7.0 & 6.3 & 6.5 & 7.4 & 7.4 & 6.9 & 6.0 & 7.5 \\
\hline 0.028 & 6.4 & 5.9 & 6.8 & 6.6 & 6.4 & 6.1 & 7.1 & 7.0 & 5.9 & 6.1 & 7.3 & 7.1 & 6.3 & 5.5 & 6.3 \\
\hline 0.056 & 6.1 & 5.1 & 5.9 & 6.5 & 6.0 & 5.6 & 7.4 & 7.0 & 5.5 & 5.1 & 6.9 & 7.4 & 6.3 & 5.4 & 5.1 \\
\hline 0.0 & 7.4 & 7.6 & 7.0 & 6.6 & 7.4 & 7.1 & 7.1 & 7.0 & 7.4 & 7.9 & 7.6 & 7.1 & 7.5 & 7.5 & 7.6 \\
\hline Trend $^{y}$ & $\mathrm{Q}$ & $\mathrm{Q}$ & $\mathrm{Q}$ & NS & $\mathrm{Q}$ & $\mathrm{Q}$ & NS & NS & $\mathrm{L}, \mathrm{Q}$ & $\mathrm{L}, \mathrm{Q}$ & NS & NS & Q & $\mathrm{L}, \mathrm{Q}$ & $\mathrm{Q}$ \\
\hline
\end{tabular}

${ }^{2}$ Turfgrass color rating scale: $1=$ completely brown, $6=$ minimally acceptable green, $9=$ dark green

${ }^{\mathrm{y}}$ Gradient trend analyses significant at $P<0.05$; NS $=$ nonsignificant; $\mathrm{L}=$ linear; $\mathrm{Q}=$ quadratic. 


\section{Turf Management}

Table 3. Bahiagrass seedhead counts at 2, 4, 6, and 8 weeks after treatment (WAT) following imidazolinone (Imazaquin, I; AC 263,222, AC) applications in May, June, and July 1992.

\begin{tabular}{|c|c|c|c|c|c|c|c|c|c|c|c|c|c|}
\hline \multirow[b]{5}{*}{ Imidazolinone } & \multirow{5}{*}{$\begin{array}{c}\text { Concn } \\
\left(\mathrm{kg} \cdot \mathrm{ha}^{-1}\right)\end{array}$} & \multicolumn{12}{|c|}{ Seedheads $/ \mathrm{m}^{2 \mathrm{z}}$} \\
\hline & & \multicolumn{12}{|c|}{ Application date } \\
\hline & & \multicolumn{4}{|c|}{19 May } & \multicolumn{4}{|c|}{26 June } & \multicolumn{4}{|c|}{23 July } \\
\hline & & & & & & & & & & & & & \\
\hline & & 2 & 4 & 6 & 8 & 2 & 4 & 6 & 8 & 2 & 4 & 6 & 8 \\
\hline $\bar{I}$ & 0.42 & $0.0^{y}$ & $0.0 \mathrm{~b}$ & $4.4 \mathrm{~b}$ & $8.6 \mathrm{~b}$ & $0.0 \mathrm{~b}$ & $0.5 \mathrm{~b}$ & $22.1 \mathrm{~b}$ & $20.8 \mathrm{~b}$ & $0.0 \mathrm{~b}$ & $0.0 \mathrm{~b}$ & $0.4 \mathrm{~b}$ & $3.0 \mathrm{~b}$ \\
\hline \multirow[t]{3}{*}{$\mathrm{AC}$} & 0.028 & --- & --- & --- & --- & $0.0 \mathrm{~b}$ & $0.0 \mathrm{~b}$ & $1.5 \mathrm{c}$ & $5.4 \mathrm{c}$ & --- & --- & --- & --- \\
\hline & 0.042 & --- & --- & --- & --- & --- & --- & --- & --- & $0.0 \mathrm{~b}$ & $0.0 \mathrm{~b}$ & $0.2 \mathrm{~b}$ & $0.3 \mathrm{~b}$ \\
\hline & 0.056 & 0.0 & $0.0 \mathrm{~b}$ & $0.0 \mathrm{~b}$ & $0.0 \mathrm{c}$ & $0.0 \mathrm{~b}$ & $0.0 \mathrm{~b}$ & $0.7 \mathrm{c}$ & $1.3 \mathrm{~cd}$ & $0.0 \mathrm{~b}$ & $0.0 \mathrm{~b}$ & $0.0 \mathrm{~b}$ & $0.0 \mathrm{~b}$ \\
\hline \multirow[t]{3}{*}{$\mathrm{I}+\mathrm{AC}$} & $0.21+0.028$ & --- & --- & --- & --- & $0.0 \mathrm{~b}$ & $0.0 \mathrm{~b}$ & $0.5 \mathrm{c}$ & $0.9 \mathrm{~cd}$ & --- & --- & --- & --- \\
\hline & $0.21+0.042$ & --- & --- & --- & --- & --- & --- & --- & --- & $0.0 \mathrm{~b}$ & $0.0 \mathrm{~b}$ & $0.0 \mathrm{~b}$ & $0.0 \mathrm{~b}$ \\
\hline & $0.21+0.056$ & 0.0 & $0.0 \mathrm{~b}$ & $0.0 \mathrm{~b}$ & $0.0 \mathrm{c}$ & $0.0 \mathrm{~b}$ & $0.0 \mathrm{~b}$ & $0.7 \mathrm{c}$ & $0.2 \mathrm{~d}$ & $0.0 \mathrm{~b}$ & $0.0 \mathrm{~b}$ & $0.0 \mathrm{~b}$ & $0.0 \mathrm{~b}$ \\
\hline Control & 0.0 & 0.0 & $1.0 \mathrm{a}$ & $20.1 \mathrm{a}$ & $30.7 \mathrm{a}$ & $12.7 \mathrm{a}$ & $43.4 \mathrm{a}$ & $44.6 \mathrm{a}$ & $44.6 \mathrm{a}$ & $4.6 \mathrm{a}$ & $15.2 \mathrm{a}$ & 20.9 a & $33.2 \mathrm{a}$ \\
\hline LSD & --- & NS & 0.8 & 8.2 & 4.9 & 1.5 & 4.3 & 10.4 & 4.5 & 4.5 & 6.6 & 5.9 & 5.2 \\
\hline
\end{tabular}

${ }^{\mathrm{z}}$ Mean seedheads $/ \mathrm{m}^{2}$ based on three random tosses of a 3- $\mathrm{dm}^{2}$ circular template within the plots.

${ }^{y}$ Mean separation within a column by Fisher's protected LSD test at $P<0.05$; nS $=$ nonsignificant.

Table 4. Bahiagrass seedhead counts at 2, 4, 6, and 8 weeks after treatment (WAT) following AC 263,222 (AC) applications in May, June, July, and Aug. 1993.

\begin{tabular}{|c|c|c|c|c|c|c|c|c|c|c|c|c|c|c|c|}
\hline \multirow{5}{*}{$\begin{array}{l}\text { AC concn } \\
\left(\mathrm{kg} \cdot \mathrm{ha}^{-1}\right)\end{array}$} & \multicolumn{15}{|c|}{ Seedheads $/ \mathrm{m}^{2 z}$} \\
\hline & \multicolumn{15}{|c|}{ Application date } \\
\hline & \multicolumn{4}{|c|}{31 May } & \multicolumn{4}{|c|}{28 June } & \multicolumn{4}{|c|}{26 July } & \multicolumn{3}{|c|}{25 Aug. } \\
\hline & & & & & & & & WAT & & & & & & & \\
\hline & 2 & 4 & 6 & 8 & 2 & 4 & 6 & 8 & 2 & 4 & 6 & 8 & 2 & 4 & 6 \\
\hline$\overline{0.014}$ & 0.0 & 1.1 & 7.5 & 15.1 & 6.5 & 11.8 & 20.4 & 45.1 & 1.1 & 2.1 & 4.3 & 3.2 & 2.2 & 1.1 & 6.5 \\
\hline 0.028 & 0.0 & 0.0 & 0.0 & 3.2 & 3.2 & 4.3 & 5.4 & 8.6 & 0.0 & 1.1 & 1.1 & 1.1 & 2.2 & 1.1 & 3.2 \\
\hline 0.056 & 0.0 & 0.0 & 0.0 & 0.0 & 2.1 & 1.1 & 1.1 & 3.2 & 0.0 & 0.0 & 0.0 & 0.0 & 1.1 & 1.1 & 1.1 \\
\hline 0.0 & 2.2 & 2.2 & 20.4 & 26.5 & 20.4 & 37.7 & 48.4 & 70.0 & 9.7 & 37.7 & 43.0 & 24.7 & 7.5 & 17.2 & 49.5 \\
\hline Trend $^{y}$ & NS & $\mathrm{Q}$ & Q & Q & $\mathrm{L}, \mathrm{Q}$ & $\mathrm{L}, \mathrm{Q}$ & $\mathrm{L}, \mathrm{Q}$ & $\mathrm{L}, \mathrm{Q}$ & $\mathrm{L}, \mathrm{Q}$ & $\mathrm{L}$ & $\mathrm{L}$ & $\mathrm{L}$ & $\mathrm{L}, \mathrm{Q}$ & $\mathrm{L}, \mathrm{Q}$ & L, Q \\
\hline
\end{tabular}

${ }^{2}$ Mean seedheads $/ \mathrm{m}^{2}$ based on three random tosses of a $3-\mathrm{dm}^{2}$ circular template within the plots

${ }^{\mathrm{y}}$ Gradient trend analyses significant at $P<0.05$; NS = nonsignificant; $\mathrm{L}=$ linear; $\mathrm{Q}=$ quadratic.

Table 5. Bahiagrass seedhead height at 2, 4, 6, and 8 weeks after treatment (WAT) following imidazolinone (Imazaquin, I; AC 263,222, AC) applications in May, June, and July 1992.

\begin{tabular}{|c|c|c|c|c|c|c|c|c|c|c|c|c|c|}
\hline \multirow[b]{5}{*}{ Imidazolinone } & \multirow{5}{*}{$\begin{array}{l}\text { Concn } \\
\left(\mathrm{kg} \cdot \mathrm{ha}^{-1}\right)\end{array}$} & \multicolumn{12}{|c|}{ Seedhead ht $(\mathrm{cm})^{\mathrm{z}}$} \\
\hline & & \multicolumn{12}{|c|}{ Application date } \\
\hline & & \multicolumn{4}{|c|}{19 May } & \multicolumn{4}{|c|}{26 June } & \multicolumn{4}{|c|}{23 July } \\
\hline & & & & & & & WA & & & & & & \\
\hline & & 2 & 4 & 6 & 8 & 2 & 4 & 6 & 8 & 2 & 4 & 6 & 8 \\
\hline I & 0.42 & $0.0^{y}$ & $0.0 \mathrm{~b}$ & $30.0 \mathrm{~b}$ & $38.7 \mathrm{~b}$ & $0.0 \mathrm{~b}$ & $0.5 \mathrm{~b}$ & $22.1 \mathrm{~b}$ & $20.8 \mathrm{~b}$ & $0.0 \mathrm{~b}$ & $0.0 \mathrm{~b}$ & $0.4 \mathrm{~b}$ & $3.0 \mathrm{~b}$ \\
\hline \multirow[t]{3}{*}{$\mathrm{AC}$} & 0.028 & --- & --- & --- & --- & $0.0 \mathrm{~b}$ & $0.0 \mathrm{~b}$ & $1.5 \mathrm{c}$ & $5.4 \mathrm{c}$ & --- & -- & --- & --- \\
\hline & 0.042 & --- & --- & --- & --- & --- & -- & --- & -- & $0.0 \mathrm{~b}$ & $0.0 \mathrm{~b}$ & $0.2 \mathrm{~b}$ & $0.3 \mathrm{~b}$ \\
\hline & 0.056 & $0.0 \mathrm{~b}$ & $0.0 \mathrm{~b}$ & $0.0 \mathrm{c}$ & $0.0 \mathrm{c}$ & $0.0 \mathrm{~b}$ & $0.0 \mathrm{~b}$ & $0.7 \mathrm{c}$ & $1.3 \mathrm{~cd}$ & $0.0 \mathrm{~b}$ & $0.0 \mathrm{~b}$ & $0.0 \mathrm{~b}$ & $0.0 \mathrm{~b}$ \\
\hline \multirow[t]{3}{*}{$\mathrm{I}+\mathrm{AC}$} & $0.21+0.028$ & --- & --- & --- & --- & $0.0 \mathrm{~b}$ & $0.0 \mathrm{~b}$ & $0.5 \mathrm{c}$ & $0.9 \mathrm{~cd}$ & --- & --- & --- & -- \\
\hline & $0.21+0.042$ & --- & --- & --- & --- & --- & --- & --- & --- & $0.0 \mathrm{~b}$ & $0.0 \mathrm{~b}$ & $0.0 \mathrm{~b}$ & $0.0 \mathrm{~b}$ \\
\hline & $0.21+0.056$ & $0.0 \mathrm{~b}$ & $0.0 \mathrm{~b}$ & $0.0 \mathrm{c}$ & $0.0 \mathrm{c}$ & $0.0 \mathrm{~b}$ & $0.0 \mathrm{~b}$ & $0.7 \mathrm{c}$ & $0.2 \mathrm{~d}$ & $0.0 \mathrm{~b}$ & $0.0 \mathrm{~b}$ & $0.0 \mathrm{~b}$ & $0.0 \mathrm{~b}$ \\
\hline Control & 0.0 & $24.0 \mathrm{a}$ & $40.0 \mathrm{a}$ & $60.0 \mathrm{a}$ & $60.0 \mathrm{a}$ & $12.7 \mathrm{a}$ & $43.4 \mathrm{a}$ & $44.6 \mathrm{a}$ & $44.6 \mathrm{a}$ & $4.6 \mathrm{a}$ & $15.2 \mathrm{a}$ & $20.9 \mathrm{a}$ & $33.2 \mathrm{a}$ \\
\hline LSD & & 13.4 & 34.7 & 2.9 & 9.8 & 1.5 & 4.3 & 10.4 & 4.5 & 4.5 & 6.6 & 5.9 & 5.2 \\
\hline
\end{tabular}

${ }^{\mathrm{z}}$ Mean seedhead height determination based on three random measurements within the plot.

yean separation within a column by Fisher's protected LSD test at $P<0.05$; NS = nonsignificant.

Table 6. Bahiagrass seedhead height at 2, 4, 6, and 8 weeks after treatment (WAT) following AC 263,222 (AC) applications in May, June, July, and Aug. 1993.

\begin{tabular}{|c|c|c|c|c|c|c|c|c|c|c|c|c|c|c|c|}
\hline \multirow{5}{*}{$\begin{array}{l}\text { AC concn } \\
\left(\mathrm{kg} \cdot \mathrm{ha}^{-1}\right)\end{array}$} & \multicolumn{15}{|c|}{ Seedhead ht $(\mathrm{cm})^{2}$} \\
\hline & \multicolumn{15}{|c|}{ Application date } \\
\hline & \multicolumn{4}{|c|}{31 May } & \multicolumn{4}{|c|}{28 June } & \multicolumn{4}{|c|}{26 July } & \multicolumn{3}{|c|}{25 Aug. } \\
\hline & & & & & & & & WAT & & & & & & & \\
\hline & 2 & 4 & 6 & 8 & 2 & 4 & 6 & 8 & 2 & 4 & 6 & 8 & 2 & 4 & 6 \\
\hline$\overline{0.014}$ & 0.0 & 18.5 & 57.0 & 66.5 & 35.0 & 43.0 & 47.5 & 73.9 & 7.5 & 21.6 & 31.4 & 51.3 & 30.5 & 35.8 & 42.0 \\
\hline 0.028 & 0.0 & 0.0 & 17.3 & 46.8 & 35.0 & 31.5 & 32.8 & 54.3 & 0.0 & 13.6 & 14.4 & 24.0 & 33.5 & 36.8 & 33.4 \\
\hline 0.056 & 0.0 & 0.0 & 0.0 & 0.0 & 34.0 & 26.0 & 31.8 & 47.6 & 0.0 & 0.0 & 0.0 & 18.8 & 28.5 & 29.3 & 26.8 \\
\hline 0.0 & 54.5 & 53.0 & 70.0 & 77.8 & 69.0 & 68.5 & 66.3 & 77.4 & 53.8 & 61.4 & 79.8 & 75.0 & 58.9 & 67.3 & 70.4 \\
\hline Trend ${ }^{y}$ & $\mathrm{~L}, \mathrm{Q}, \mathrm{C}$ & $\mathrm{L}, \mathrm{Q}$ & $\mathrm{Q}, \mathrm{C}$ & $\mathrm{Q}, \mathrm{C}$ & $\mathrm{L}, \mathrm{Q}$ & $\mathrm{L}, \mathrm{Q}, \mathrm{C}$ & $\mathrm{L}, \mathrm{Q}$ & Q & $\mathrm{L}, \mathrm{Q}, \mathrm{C}$ & $\mathrm{L}, \mathrm{Q}, \mathrm{C}$ & $\mathrm{L}, \mathrm{Q}$ & Q & $\mathrm{L}, \mathrm{Q}, \mathrm{C}$ & $\mathrm{L}, \mathrm{Q}, \mathrm{C}$ & L, Q \\
\hline
\end{tabular}

${ }^{z}$ Mean seedhead height averaged across three random measurements within the plots.

${ }^{\mathrm{y}}$ Gradient trend analyses significant at $P<0.05$; NS = nonsignificant; $\mathrm{L}=$ linear; $\mathrm{Q}=$ quadratic; $\mathrm{C}=$ cubic. 
Table 7. Bahiagrass vegetative canopy height at 2, 4, 6, and 8 weeks after treatment (WAT) following imidazolinone (Imazaquin, I; AC 263,222, AC) applications in May, June, and July 1992.

\begin{tabular}{|c|c|c|c|c|c|c|c|c|c|c|c|c|c|}
\hline \multirow[b]{5}{*}{ Imidazolinone } & \multirow{5}{*}{$\begin{array}{c}\text { Concn } \\
\left(\mathrm{kg} \cdot \mathrm{ha}^{-1}\right)\end{array}$} & \multicolumn{12}{|c|}{ Canopy ht $(\mathrm{cm})^{2}$} \\
\hline & & \multicolumn{12}{|c|}{ Application date } \\
\hline & & \multicolumn{4}{|c|}{19 May } & \multicolumn{4}{|c|}{26 June } & \multicolumn{4}{|c|}{23 July } \\
\hline & & & & & & & WA & & & & & & \\
\hline & & 2 & 4 & 6 & 8 & 2 & 4 & 6 & 8 & 2 & 4 & 6 & 8 \\
\hline I & 0.42 & $10.0 \mathrm{~b}^{\mathrm{y}}$ & $11.7 \mathrm{~b}$ & $22.5 \mathrm{a}$ & $26.0 \mathrm{ab}$ & $9.0 \mathrm{~b}$ & $14.0 \mathrm{~b}$ & $30.7 \mathrm{ab}$ & $29.3 \mathrm{ab}$ & $9.2 \mathrm{~b}$ & $8.7 \mathrm{bc}$ & $14.3 \mathrm{~b}$ & $23.5 \mathrm{a}$ \\
\hline \multirow[t]{3}{*}{ AC } & 0.028 & --- & --- & --- & --- & $10.0 \mathrm{~b}$ & $10.0 \mathrm{c}$ & $23.3 \mathrm{ab}$ & $34.3 \mathrm{ab}$ & -- & --- & --- & --- \\
\hline & 0.042 & --- & --- & --- & --- & --- & --- & --- & --- & $8.7 \mathrm{~b}$ & $9.8 \mathrm{~b}$ & $12.7 \mathrm{bc}$ & $16.0 \mathrm{~b}$ \\
\hline & 0.056 & $9.5 \mathrm{~b}$ & $10.3 \mathrm{~b}$ & $15.2 \mathrm{~b}$ & $19.5 \mathrm{~b}$ & $10.0 \mathrm{~b}$ & $9.8 \mathrm{c}$ & $17.0 \mathrm{~b}$ & $26.3 \mathrm{~b}$ & $9.2 \mathrm{~b}$ & $9.0 \mathrm{bc}$ & $10.5 \mathrm{bc}$ & $10.2 \mathrm{~b}$ \\
\hline \multirow[t]{3}{*}{$\mathrm{I}+\mathrm{AC}$} & $0.21+0.028$ & --- & --- & --- & --- & $9.2 \mathrm{~b}$ & $9.5 \mathrm{c}$ & $24.0 \mathrm{ab}$ & $29.0 \mathrm{~b}$ & --- & --- & --- & --- \\
\hline & $0.21+0.042$ & --- & --- & --- & --- & --- & --- & --- & --- & $9.0 \mathrm{~b}$ & $8.8 \mathrm{bc}$ & $10.2 \mathrm{bc}$ & $11.8 \mathrm{~b}$ \\
\hline & $0.21+0.056$ & $9.5 \mathrm{~b}$ & $10.2 \mathrm{~b}$ & $13.2 \mathrm{~b}$ & $19.0 \mathrm{~b}$ & $8.7 \mathrm{~b}$ & $9.3 \mathrm{c}$ & $16.8 \mathrm{~b}$ & $27.3 \mathrm{~b}$ & $9.3 \mathrm{~b}$ & $8.3 \mathrm{c}$ & $9.3 \mathrm{c}$ & $10.3 \mathrm{~b}$ \\
\hline Control & 0.0 & $16.5 \mathrm{a}$ & $23.8 \mathrm{a}$ & $27.3 \mathrm{a}$ & $32.3 \mathrm{a}$ & $14.8 \mathrm{a}$ & $23.7 \mathrm{a}$ & $26.0 \mathrm{a}$ & $39.0 \mathrm{a}$ & $15.0 \mathrm{a}$ & $23.3 \mathrm{a}$ & $24.7 \mathrm{a}$ & $24.7 \mathrm{a}$ \\
\hline LSD & & 2.9 & 4.9 & 7.3 & 11.2 & 2.7 & 2.3 & 7.7 & 9.9 & 1.5 & 1.4 & 4.6 & 6.6 \\
\hline
\end{tabular}

${ }^{\mathrm{z}}$ Mean seedhead height determination based on three random measurements within the plot.

${ }^{y}$ Means within a column followed by the same letter are not significantly different according to Fisher's protected LSD test at $P<0.05$.

Table 8. Bahiagrass vegetative canopy height at 2, 4, 6, and 8 weeks after treatment (WAT) following AC 263,222 (AC) applications in May, June, July, and Aug. 1993.

\begin{tabular}{|c|c|c|c|c|c|c|c|c|c|c|c|c|c|c|c|}
\hline \multirow{5}{*}{$\begin{array}{l}\text { AC concn } \\
\left(\mathrm{kg} \cdot \mathrm{ha}^{-1}\right)\end{array}$} & \multicolumn{15}{|c|}{ Canopy ht $(\mathrm{cm})^{\mathrm{z}}$} \\
\hline & \multicolumn{15}{|c|}{ Application date } \\
\hline & \multicolumn{4}{|c|}{31 May } & \multicolumn{4}{|c|}{28 June } & \multicolumn{4}{|c|}{ 26 July } & \multicolumn{3}{|c|}{25 Aug. } \\
\hline & & & & & & & & $\overline{\text { WAT }}$ & & & & & & & \\
\hline & 2 & 4 & 6 & 8 & 2 & 4 & 6 & 8 & 2 & 4 & 6 & 8 & 2 & 4 & 6 \\
\hline 0.014 & 24.5 & 23.3 & 40.0 & 43.5 & 30.5 & 33.8 & 34.8 & 42.5 & 21.5 & 25.9 & 34.1 & 32.0 & 21.9 & 22.8 & 20.0 \\
\hline 0.028 & 25.3 & 22.5 & 33.5 & 40.8 & 29.3 & 27.3 & 31.8 & 41.3 & 18.5 & 24.4 & 30.4 & 31.5 & 22.5 & 20.8 & 20.0 \\
\hline 0.056 & 23.5 & 21.3 & 27.1 & 34.8 & 28.3 & 25.5 & 29.7 & 36.9 & 18.5 & 18.8 & 28.5 & 28.3 & 19.4 & 19.0 & 16.3 \\
\hline 0.0 & 31.8 & 29.5 & 43.3 & 49.8 & 40.0 & 45.0 & 40.3 & 47.1 & 32.3 & 39.3 & 45.9 & 37.0 & 33.3 & 29.0 & 31.0 \\
\hline Trend $^{y}$ & NS & Q & $\mathrm{Q}$ & $\mathrm{Q}$ & NS & $\mathrm{Q}$ & NS & $\mathrm{Q}$ & $\mathrm{L}, \mathrm{Q}, \mathrm{C}$ & $\mathrm{L}, \mathrm{Q}, \mathrm{C}$ & $\mathrm{Q}$ & NS & $\mathrm{L}, \mathrm{Q}$ & $\mathrm{Q}$ & $\mathrm{L}, \mathrm{Q}$ \\
\hline
\end{tabular}

${ }^{2}$ Mean vegetative canopy height averaged across three random measurements within the plots.

${ }^{\mathrm{y}}$ Gradient trend analyses significant at $P<0.05$; NS $=$ nonsignificant; $\mathrm{L}=$ linear; $\mathrm{Q}=$ quadratic; $\mathrm{C}=$ cubic.

pared to the control 4 WAT in 1992 (Table 7). However, the reductions were not different from the nontreated control by 6 WAT for the May and June applications, and by 8 WAT for the July application. All treatments containing $\mathrm{AC}$ at $0.056 \mathrm{~kg} \cdot \mathrm{ha}^{-1}$ in 1992 reduced vegetative canopy height at least $33 \%$ by 8 WAT (Table 7).

Quadratic trends in vegetative canopy height for 1993 were obtained for AC treatments from 4 through 8 WAT following May applications, at 4 and 8 WAT following June, and through 6 WAT following July and $\mathrm{Au}-$ gust applications (Table 8). The differences in vegetative canopy height following $\mathrm{AC}$ treatment, although significant for most months, were not as pronounced as observed for seedhead count and seedhead height reductions. Imidazolinones also provided varying degrees of vegetative and reproductive growth suppression of common bermudagrass (Cynodon dactylon L. Pers.) (Goatley et al., 1993).

At the concentrations used in these trials, imazaquin was not as effective in growth regulation of bahiagrass as AC, although imazaquin generally resulted in less turf discoloration. Combinations of imazaquin and $\mathrm{AC}$ did not increase activity over the same levels of AC applied alone.

AC provided excellent bahiagrass seedhead control up to $8 \mathrm{WAT}$, depending on timing and level of the application. The 0.042- and 0.056$\mathrm{kg} \cdot \mathrm{ha}^{-1}$ rates were generally necessary for total seedhead control through 8 WAT in periods of rapid bahiagrass growth (usually during the months of May and June in Mississippi). AC at
$0.056 \mathrm{~kg} \cdot \mathrm{ha}^{-1}$ severely discolored bahiagrass through 8 WAT following July 1992 and Aug. 1993 applications. Rates of 0.014 and 0.028 $\mathrm{kg} \cdot \mathrm{ha}^{-1}$ provided nearly $100 \%$ seedhead control following July or August applications in 1993. This finding indicates that AC at 0.014 or $0.028 \mathrm{~kg} \cdot \mathrm{ha}^{-1}$ may provide acceptable growth regulation during periods when the bahiagrass growth is slowed by possible moisture stress conditions resulting from limited summer rainfall. Under these environmental conditions, the lower concentrations of AC also resulted in significantly less turf discoloration than the $0.042-$ or $0.056-\mathrm{kg} \cdot \mathrm{ha}^{-1}$ concentrations.

Turf discoloration following AC application warrants concern. Much of the discoloration observed in these trials would be acceptable for highway rights-of-way, but may not be acceptable for improved turfgrass areas. Chemical application programs currently used for bahiagrass growth regulation generally provide either 1) relatively short periods of bahiagrass growth regulation (up to 4 weeks), but minimal turfgrass phytotoxicity (Flanagan and Peacock, 1986) or 2) seedhead control up to $10 \mathrm{WAT}$, but severe bahiagrass phytotoxicity through 4 to 6 WAT (Johnson, 1990). The superior efficacy of AC for bahiagrass seedhead control is promising for the development of a chemical application program that will provide at least 8 weeks of seedhead control, while possibly reducing turfgrass phytotoxicity by using lower rates of AC during periods of slower bahiagrass growth. Further research is necessary to determine precise levels and timings of AC applications, and to ascertain how the chemical applications can be inte- grated with mowing programs for bahiagrass turf while maintaining acceptable levels of growth regulation and turf discoloration.

\section{Literature Cited}

Beard, J.B. 1973. Turfgrass: Science and culture. Prentice Hall, Englewood Cliffs, N.J. p. 154 156.

Busey, P. 1985. Selection of seedhead characteristics in bahiagrass. Agron. Abstr. Amer. Soc. Agron., Madison, Wis. p. 114.

DiPaola, J.M., W.M. Lewis, and W.B. Gilbert. 1985. Roadside growth retardant research update. Proc. 23rd Annu. North Carolina Turfgrass Conf., p. 41-43. North Carolina State Univ., Raleigh.

Flanagan, M.S. and C.H. Peacock. 1986. Effects of plant growth regulators on bahiagrass. Turfgrass Research in Florida. Tech. Rpt., p. 67-72. Univ. of Florida, Gainesville.

Goatley, J.M., Jr., V.L. Maddox, and R.M. Watkins. 1993. Growth regulation of common bermudagrass (Cynodon dactylon) with imazaquin and AC 263,222. Weed Technol. 7:746-750.

Johnson, B.J. 1990. Response of bahiagrass (Paspalum notatum) to plant growth regulators. Weed Technol. 4:895-899.

Lentner, M. and T. Bishop. 1986. Experimental design and analysis. Valley Book Co., Blacksburg, Va. p. 107-136.

McCarty, L.B., D.L. Colvin, and R.D. Baker. 1993. Sequential year application of PGR's for bahiagrass seedhead suppression. Proc. Southern Weed Sci. Soc. 46:232.

SAS Institute. 1987. SAS user's guide: Statistics. SAS Inst., Cary, N.C.

Watschke, T.L., M.G. Prinster, and J.M. Breuninger. 1992. Plant growth regulators and turfgrass management, p. 558-588. In: D.V. Waddington, R.N. Carrow, and R.C. Shearman (eds.). Turfgrass. Amer. Soc. Agron., Madison, Wis. 